\title{
Interactive Sonification of Curve Shape and Curvature Data
}

\author{
Simon Shelley ${ }^{1}$, Miguel Alonso ${ }^{1}$. Jacqueline Hollowood ${ }^{2}$, Michael Pettitt ${ }^{2}$, \\ Sarah Sharples ${ }^{2}$, Dik Hermes ${ }^{1}$, and Armin Kohlrausch ${ }^{1}$ \\ 1 Human Technology Interaction, Eindhoven University of Technology, P.O. Box 513, \\ NL 5600 MB, Eindhoven, The Netherlands \\ d.j.hermes@tue.nl \\ ${ }^{2}$ Human Factors Research Group, The University of Nottingham, University Park, \\ Nottingham, NG7 2RD UK
}

\begin{abstract}
This paper presents a number of different sonification approaches that aim to communicate geometrical data, specifically curve shape and curvature information, of virtual 3-D objects. The system described here is part of a multi-modal augmented reality environment in which users interact with virtual models through the modalities vision, hearing and touch. An experiment designed to assess the performance of the sonification strategies is described and the key findings are presented and discussed.
\end{abstract}

Keywords: sonification, sound synthesis, modal synthesis, virtual environments, haptics, human-computer interaction.

\section{Introduction}

The work presented in this paper describes how sound is used in a multimodal design environment in the context of the European project called: Sound And Tangible Interfaces for Novel product design (SATIN). The aim of the project is to develop a multimodal interface consisting of an augmented reality environment in which a product designer is able to see virtual 3-D objects and to both explore and modify the shape of these objects by directly touching them with his/her hands. This visual-haptic interface is supplemented by the use of sound as a means to convey information and feedback about the virtual object and the user interaction. More specifically, the use of sonification allows the designer to explore geometric properties related to the surface of the object that are hardly detectable by touch or sight.

The sonification of the surface shape and curvature of the virtual object is a specific requirement of the SATIN project. Shape and curvature data are of special interest to designers when considering the aesthetic quality of the object's surface. In industrial design, for example, a smooth surface that is continuous in terms of curvature is highly desirable because discontinuities in curvature result in a disconnected appearance in the light reflected from the surface. Designers 
refer to surfaces with continuous curvature as Class A surfaces and they consider them as being aesthetically appealing [1].

This article is organized as follows. Section 2 of this paper presents a short summary of work related to this study. Then in section 3 we propose and describe the sonification strategies. This is followed by section 4 where the evaluation and results are presented. Finally, a summary of the work and a discussion of the results are given in section 5 ,

\section{Related Work}

Previous work involved physical models that are able to synthesize sounds that are perceptually associated with interactions between humans and physical objects 2[3/4. In studies involving interaction with virtual objects this type of auditory feedback can be used to convey important environmental information in order to improve perception and performance [5]6].

One of the requirements of the SATIN project is the interactive sonification of numerical data related to the geometrical properties of a virtual object. Another related study suggests the use of sonification as a means to present geometrical data of surfaces in scientific and engineering applications [7]. However, so far in this context we are not aware of any study carrying out a formal evaluation of the success of such an approach.

In the current literature there exists a number of studies with a similar aim, but in a variety of different contexts. For example, some attempts have been made to sonify numerical data for people with visual impairment; in some cases this is combined and/or compared with kinaesthetic methods used to represent the same data 8191011]. Perhaps one of the earliest studies was made by Mansur who used a sinusoidal wave, the frequency of which is used to sonify the ordinate value of a numerical function where the abscissa is mapped to time. Mansur discovered that mathematical concepts such as symmetry, monotonicity and the slopes of lines could be determined using sound after a relatively small amount of training [10]. Another related application domain involves the sonification of financial data 12 1314. The aim of such systems is to inform the user about financial information, for example stock market prices.

Recently, researchers have also been interested in a more general approach to sonification, in which the aim is to produce sonification toolkits. Such toolkits are designed to allow users to map arbitrary data to sound with relative ease. Examples of sonification toolkits include Listen [15] and Muse [16], developed by Lodha, Musart [17, developed by Joseph and Lodha and the Sonification Sandbox [18, developed by Walker and Cothran. However, these toolkits are not suitable for the SATIN project because, in their current state, they rely on pre-defined data sets. In the case of the SATIN project the data are produced dynamically by the interaction of the user with other elements of the system. 

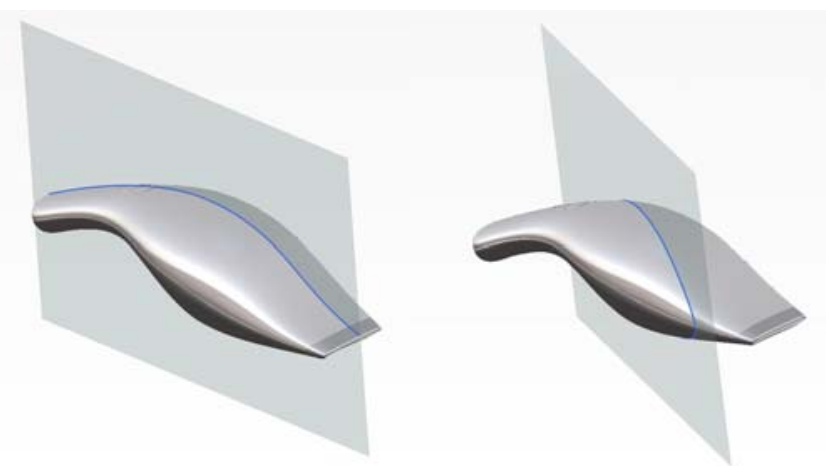

Fig. 1. Example of a virtual 3-D object with two potential 2-D cross-sectional slices highlighted

\section{Sonification of Geometrical Data}

In the SATIN system, although dealing with 3-D models, the haptic interaction is limited to a 2-D cross-sectional slice of the virtual object's surface. The user is free to select this slice from any part of the 3-D object. An example of two different cross-sections of the same 3-D virtual object is illustrated in Fig. 1. In this work we consider two specific requirements of the SATIN project, which are the sonification of data relating to the curve shape and curvature of the surface of the virtual object along the cross-sectional plane of interest. It is important to note that curve shape and curvature information are not sonified simultaneously, but prior to the operation of the system the user can select which of these two geometrical properties will be sonified. The aim of the sonification system is to inform the user about the chosen geometrical property of interest at the point of contact between the user's finger and the haptic device. The system is designed so that only one point of contact along the 2-D cross section can be evaluated using sound at any one time. The data to be sonified are described below.

- Curve shape: We consider only a 2-D cross-sectional slice of the virtual object's surface such as those highlighted in Fig. 1. As a result, the curve shape is a plane curve $C$ represented by a two-dimensional function. For example, an arbitrarily generated curve shape is shown in Fig. 2, which illustrates the shape of an object's surface along the cutting plane.

- Curvature: For a given plane curve $C$, the curvature at point $p$ has a magnitude equal to the reciprocal of the radius of the osculating circle, i.e. the circle that shares a common tangent to the curve at the point of contact. The curvature is a vector that points to the centre of the osculating circle. For a plane curve given explicitly as $C=f(p)$ the curvature is given by:

$$
\mathcal{K}=\frac{\frac{d^{2} C}{d p^{2}}}{\left(1+\left[\frac{d C}{d p}\right]^{2}\right)^{\frac{3}{2}}}
$$




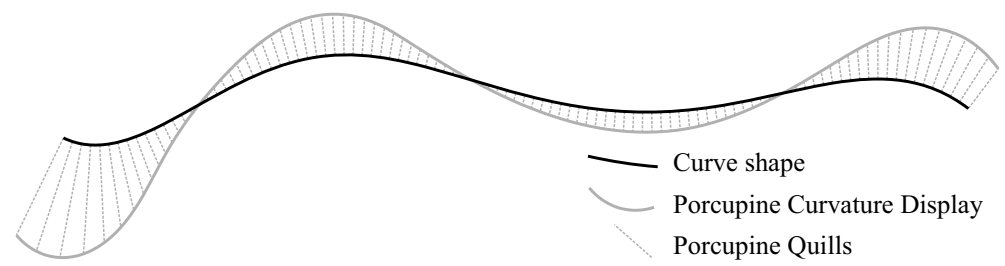

Fig. 2. Illustration of curve shape with curvature illustrated using a porcupine display

In Fig. 2 the curvature of the afore mentioned curve shape is illustrated by using a technique referred to as porcupine display. Porcupine quills are perpendicular to the curve and their lengths are proportional to the curvature at the point of contact with the curve shape, as given by (1). Therefore the longer the porcupine quill line, the higher the value of curvature, i.e. the tighter the curve. Note that in this example if the porcupine quill is drawn on top of the curve shape this denotes a negative curvature value, i.e. a convex shape, whereas a porcupine drawn underneath the curve shape denotes a positive curvature value, i.e. a concave shape.

\section{Modes of interaction}

During the sonification of the curve shape and curvature of a virtual object, three different modes of haptic interaction are considered, as illustrated in Fig. 3. The first involves the impact between the user's finger and the model at a certain point, for example by tapping the object, as shown in Fig. 3(a). The second, Fig. 3(b), involves a sustained contact with the object without movement. Finally, the third type of interaction, Fig. 3(c), involves sliding a finger over the object.

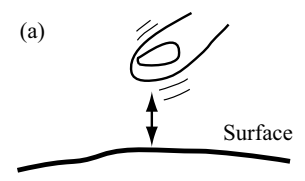

(b)

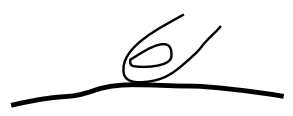

(c)

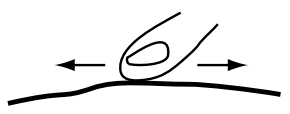

Fig. 3. Modes of user interaction with the virtual model

\subsection{Sonification Approaches}

The selection of sound synthesis approaches and mapping strategies to sonify the geometrical data of interest may have a significant effect on how the information is perceived by the user. The users expressed a wish for the sound that is produced to be influenced by the mode of interaction in an intuitive way. In an attempt to address this, we developed a post-processing module which we refer to in this article as the kinetic module. This module post-processes the generated sound according to the interaction between the user's fingers and the haptic device, i.e., the speed of the finger and pressure it exerts on the device. Furthermore, three different sonification strategies were designed. These strategies and the kinetic module are described later in the text. 
As the final haptic interface is in development, we are currently using a digital drawing tablet as an alternative input device. Contact between the drawing tablet pen and the tablet is analogous to contact between the user's finger and the haptic device in the SATIN project. The position of contact along the $x$-axis of the drawing tablet is equivalent to the position of finger contact along the length of the selected cross-sectional profile represented by the haptic interface. In order to develop and prototype the sonification platform, we use the Max/MSP graphical development environment.

\subsection{Mapping Strategy}

In each of the three sonification methods, the value of the geometrical parameter of interest, curve shape or curvature, is mapped to the fundamental frequency of the carrier sound. The mapping is implemented in such a way that the minimum absolute value of the geometrical parameter is mapped to a minimum frequency of $200 \mathrm{~Hz}$ and the maximum absolute value found in the dataset is mapped to a maximum frequency of $800 \mathrm{~Hz}$. Additionally, linear changes in the geometrical parameter of interest are mapped to logarithmic changes in the sound carrier frequency. After experimenting with a number of mappings, users considered this to be the easiest to understand. In fact, frequency is a common choice for this type of application and it is considered to be particularly strong as a means to sonify changing values [19]. Humans perceive frequency changes with a relatively high resolution. Typically, frequency changes of pure tones can be perceived with an accuracy of up to $0.3 \%$ [20]. The following sub-sections describe the three sonification strategies used in this study, named after the sound synthesis technique used to sonify or carry the information of interest.

\subsection{Sinusoidal Carrier}

For this approach, a pure sinusoidal tone is used as the carrier and its frequency is modulated according to the mapping strategy described in the previous subsections.

\subsection{Wavetable Sampling Carrier}

For this strategy, a continuous sound is produced whilst the user remains in contact with the haptic device. This sound is made up of a harmonically rich recording of a bowed cello. The attack and decay parts of the note are removed from the sound excerpt, leaving only the middle part with a stable fundamental frequency. The fundamental frequency of the recorded note is $131 \mathrm{~Hz}$, which closely corresponds to C3 on the Western musical scale. This sound is continuously played back in a loop, in such a way that no audible artefacts or interruptions are present.

Given the harmonically rich nature of the cello sound, the technique referred to as Time-Domain Pitch-Synchronous Overlap-Add (TD-PSOLA) provides a high quality yet computationally efficient way to modify the fundamental frequency 
of the sound [21]. Using this technique, the fundamental frequency of the cello sound is mapped to the geometrical property of interest according to the mapping strategy defined in sub-section 3.2 .

\subsection{Physical Modelling Carrier}

In this case the carrier sound is generated using a modal synthesis approach. Modal synthesis is a physical modelling technique for sound rendering, with theoretical roots in modal analysis [22]. The aim of modal synthesis is to mimic the dynamic properties of an elastic structure in terms of its characteristic modes of vibration. The modal synthesis implementation employed for this work is based on research by Van den Doel and Pai [23. A more detailed explanation of this approach and its implementation in the SATIN project is given in 24].

For this study, a model of a circular plate was implemented, resulting in a sound with a rich but in-harmonic spectrum. The modal frequencies of the plate were calculated by solving the wave equation for a circular plate [25. In order to sonify the geometrical data we map the magnitude of the parameter of interest to the fundamental frequency of the modal synthesis model, according to the mapping strategy described in sub-section 3.2. The perceptual effect of frequency scaling in this way gives the impression that, as the fundamental frequency increases, the modelled object decreases in size.

\subsection{Kinetic Module}

The kinetic module is an optional addition to the sonification system that postprocesses the generated sound according to the interaction between the user's fingers and the haptic device. When the kinetic module is switched on, the intensity and the spectral tilt of the selected sound are varied according to the pressure and exploration speed exerted by the user's finger. In addition, the sound is passed through a high-pass filter with a cut-off frequency that is varied according to the pressure of the user's finger. This technique is based on a perceptual study, the details of which can be found in [26]. Finally, with the kinetic module switched on, if the user taps the haptic interface, as illustrated by Fig. 3(a), a short impulse of the selected sound is produced. Note that, apart from varying its intensity, the kinetic module has very little perceptual effect on the sinusoidal carrier, which consists only of a single spectral component.

\section{Evaluation and Results}

This paper presents a selection of results from two separate studies, performed as part of a wider experiment aimed at investigating the use of sound in the SATIN system [27]. These studies were designed to evaluate the performance of the sonification strategies as a means to communicate both curve shape and curvature. Twelve test subjects participated in the evaluation, with the requirement that they all have a basic understanding of calculus. In both studies all three of the sound carriers described above were used and compared. In the case of 


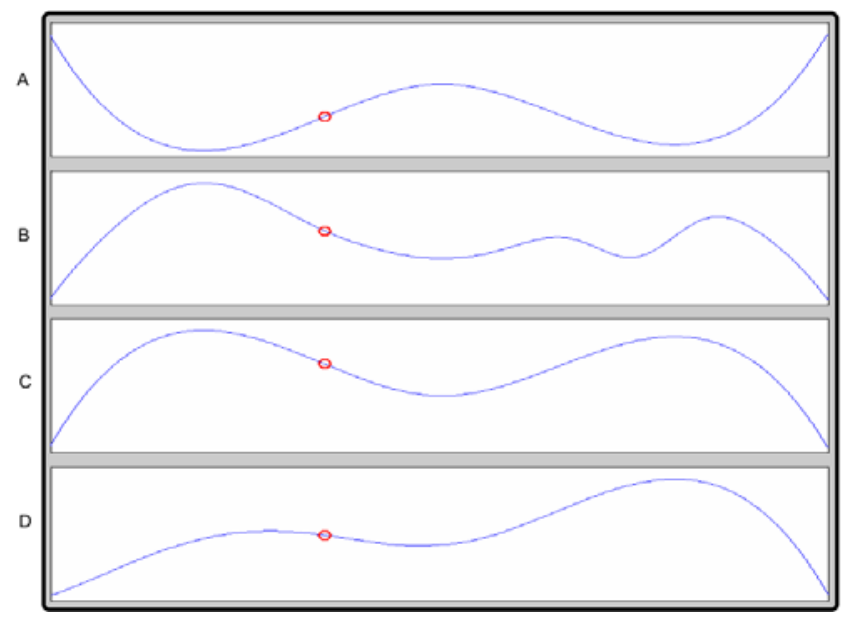

Fig. 4. Screen-shot showing an example of the multiple choice options presented to the test subjects. The small circle indicates the position on the curve that the user is exploring at that time.

wavetable sampling carrier and the physical modelling carrier we also tested the effect of applying the kinetic module. For the first experiment a number of different synthetic curve shapes were generated. The subjects were invited to explore the curve shape using a stylus on a digital drawing tablet as input, as described earlier. At the same time they were presented with the graphical representations of four different curve shapes, of which only one corresponds correctly to the sonified data. From these options, they were then asked to choose which of the curves best corresponds to the sounds that resulted from their exploration using the stylus. Fig. 4 is a screen-shot showing an example of the multiple choice options presented to the test subjects. The circle is used to convey to the test subject the position on the curve that he/she is exploring at that time.

For the second experiment, the same group of subjects were invited to another session, in which they were again presented with the graphical representations of four curve shapes. This time the data sonified corresponds to the curvature of one of the presented curve shapes. As an example, for the curve illustrated in Fig. 2, the subject would see the black curve but would listen to the sonification of the grey curve. In this case the subjects were given a short explanation of how curvature is related to curve shape.

Fig. [5 shows the average percentage of correct answers and response times for each sonification strategy and for both experiments. A significant finding is that each of these strategies, both with and without the kinetic module, performed at an equivalent level of about $80 \%$ accuracy. In addition, as a result from questionnaires administered during the tests, it was discovered that the subjects exhibited a preference for the sinusoidal and wavetable sampling carrier approaches. It was also found that participants needed in the region of 30 seconds to make their decision in each of the multiple choice questions. 

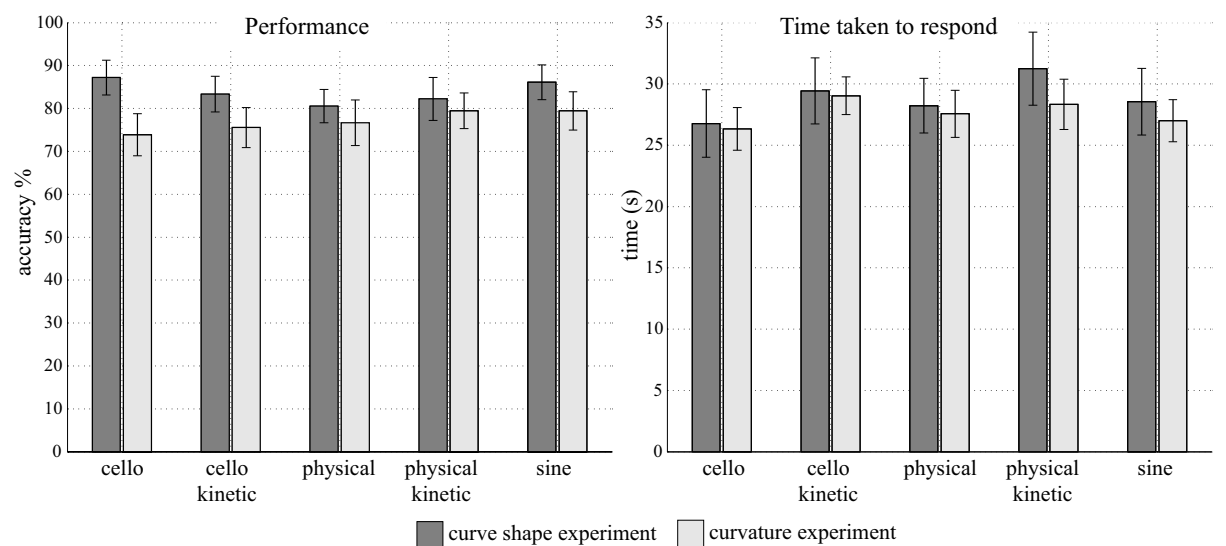

Fig. 5. Judgement accuracy and response time for both curve shape and curvature experiments

\section{Conclusions}

In this paper we present a sonification approach to communicate information about the curve shape and curvature of a virtual 3 -D object. The described auditory system is part of a multi-modal augmented reality environment where designers can interact with 3-D models through the additional modalities of vision and touch. We implemented three sonification approaches that share a common mapping scheme, in which the parameter to sonify is mapped to the fundamental frequency, or the set of modal frequencies, of the sound carrier. The three approaches consisted of sinusoidal, wavetable sampling and physical modelling sound carriers. In addition, a novel concept is presented in which the sound that is produced is intuitively influenced by the way that the user explores the virtual object. A post-processing module, referred to in this article as the kinetic module, has been developed to implement this concept. A number of experiments have been conducted in order to evaluate the performance of these sonification strategies in communicating both curve shape and curvature information. The results, for a set of twelve participants, show a consistently high level of performance in all cases of about $80 \%$ accuracy for both curvature and curve shape. Participants required on average around 30 seconds to choose their answer in the multiple choice test. Finally, in this specific implementation and context, we could not find any advantage of using the kinetic module either in terms of performance or user preference.

The main contribution of this work is to show the feasibility of communicating geometrical data through sonification. We have attempted to validate this concept by means of a formal evaluation. We consider the obtained results to be highly encouraging since they suggest that humans, previously unexposed to the system, are capable of establishing a clear connection between the sound and the underlying data. This relatively high level of performance is consistent 
for the curvature experiment. We consider this to be a remarkable result, as in this case the sound is related to one of the presented images through the nontrivial mathematical expression given by (11), rather than the direct relationship exhibited in the curve shape experiment.

\section{Acknowledgments}

This research work has been partially supported by the European Commission under the project FP6-IST-5-054525 SATIN - Sound And Tangible Interfaces for Novel product design and by the COST Action IC0601 on Sonic Interaction Design. The authors also wish to thank Patrick Bosinco for providing Fig. 1 .

\section{References}

1. Catalano, C.A., Falcidieno, B., Giannini, F., Monti, M.: A survey of computeraided modeling tools for aesthetic design. Journal of Computing and Information Science in Engineering 2, 11-20 (2002)

2. Rocchesso, D., Fontana, F. (eds.): The Sounding Object. Mondo Estremo (2003)

3. Van den Doel, K., Kry, P.G., Pai, D.K.: Foleyautomatic: Physically-based sound effects for interactive simulation and animation. In: Proceedings of ACM SIGGRAPH, Los Angeles, CA, USA, August 2001, pp. 12-17 (2001)

4. Van den Doel, K., Pai, D.K.: The sounds of physical shapes. Presence 7(4), 382-395 (1998)

5. Díaz, I., Hernantes, J., Mansa, I., Lozano, A., Borro, D., Gil, J., Sánchez, E.: Influence of multisensory feedback on haptic accessibility tasks. Virtual Reality 10, 31-40 (2006); Special Issue on Multisensory Interaction in Virtual Environments

6. Kjœr, H.P., Taylor, C.C., Serafin, S.: Influence of interactive auditory feedback on the haptic perception of virtual objects. In: Proceedings of the 2nd International Workshop on Interactive Sonification, New York, UK (2007)

7. Minghim, R., Forrest, A.R.: An illustrated analysis of sonification for scientific visualisation. In: Proceedings of the 6th IEEE Visualization Conference, pp. 110-117 (1995)

8. Kennel, A.R.: Audiograf: a diagram-reader for the blind. In: Proceedings of the second annual ACM conference on Assistive technologies, pp. 51-56 (1996)

9. Stevens, R.D., Edwards, A.D.N., Harling, P.A.: Access to mathematics for visually disabled students through multimodal interaction. Human-Computer Interaction 12(1), 47-92 (1997)

10. Mansur, D.L., Blattner, M.M., Joy, K.I.: Sound graphs: A numerical data analysis method for the blind. Journal of Medical Systems 9, 163-174 (1985)

11. Roth, P., Kamel, H., Petrucci, L., Pun, T.: A comparison of three nonvisual methods for presenting scientific graphs. Journal of Visual Impairment \& Blindness 96(6), 420-428 (2002)

12. Mezrich, J., Frysinger, S., Slivjanovski, R.: Dynamic representation of multivariate time series data. Journal of the American Statistical Association 79(385), 34-40 (1984)

13. Janata, P., Childs, E.: Marketbuzz: Sonification of real-time financial data. In: Proceedings of the 10th International Conference on Auditory Display, Sydney, Australia, July 6-9 (2004) 
14. Mauney, B.S., Walker, B.N.: Creating functional and livable soundscapes for peripheral monitoring of dynamic data. In: Proceedings of the 10th International Conference on Auditory Display, Sydney, Australia, July 6-9 (2004)

15. Wilson, C.M., Lodha, S.K.: Listen: A data sonification toolkit. In: Proceedings of the 3rd International Confonference on Auditory Display, Palo Alto, USA (1996)

16. Lodha, S.K., Beahan, J., Heppe, T., Joseph, A.J., Zne-Ulman, B.: Muse: A musical data sonificaton toolkit. In: Proceedings of the 4th International Confonference on Auditory Display, Palo Alto, USA (1997)

17. Joseph, A.J., Lodha, S.K.: Musart: Musical audio transfer function real-time toolkit. In: Proceedings of the 8th International Conference on Auditory Display, Kyoto, Japan (2002)

18. Walker, B.N., Cothran, J.T.: Sonification sandbox: A graphical toolkit for auditory graphs. In: Proceedings of the 9th International Conference on Auditory Display, Boston, MA, USA (2003)

19. Stockman, T., Nickerson, L.V., Hind, G.: Auditory graphs: A summary of current experience and towards a research agenda. In: Proceedings of the 11th International Conference on Auditory Display, Limerick, Ireland (July 2005)

20. Wier, C.C., Jesteadt, W., Green, D.M.: Frequency discrimination as a function of frequency and sensation level. Journal of the Acoustical Society of America 61, 178-183 (1977)

21. Moulines, E., Charpentier, F.: Pitch-synchronous waveform processing techniques for text-to-speech synthesis using diphones. Speech Communication 9(5/6), 453-467 (1990)

22. Rossing, T.D., Fletcher, N.H.: Principles of Vibration and Sound, 2nd edn. Springer, Heidelberg (2004)

23. Van den Doel, K., Pai, D.K.: Modal Synthesis for Vibrating Objects. In: Audio anecdotes: tools, tips, and techniques for digital audio. AK Press (2004)

24. Alonso, M., Shelley, S., Hermes, D., Kohlrausch, A.: Evaluating geometrical properties of virtual shapes using interactive sonification. In: IEEE International Workshop on Haptic Audio visual Environments and Games, pp. 154-159 (2008)

25. Leissa, A.W.: Vibration of Plates, NASA SP-160. NASA, Washington (1969)

26. Hermes, D.J., van der Pol, K., Vankan, A., Boeren, F., Kuip, O.: Perception of rubbing sounds. In: Proceedings of the International Workshop on Haptic and Audio Interaction Design, Jyväskylä, Finland (2008)

27. Hollowood, J., Pettitt, M., Shelley, S.B., Alonso, M.A., Sharples, S., Hermes, D.: Results of investigation into use of sound for curvature and curve shape perception. Technical report, internal deliverable to SATIN project FP6-IST-5-054525 (2009) 